\title{
Ultra-Low Power Data Converters with BEOL NEM Relays
}

\author{
Ren Li and Hossein Fariborzi \\ Computer, Electrical and Mathematical Sciences and Engineering Division \\ King Abdullah University of Science and Technology, Thuwal 23955-6900, Saudi Arabia \\ Email: \{ren.li,hossein.fariborzi\}@kaust.edu.sa
}

\begin{abstract}
With the fundamental limitation on transistor scaling and energy efficiency, Nano-electromechanical (NEM) relays have emerged as a promising alternative solution for ultralow power integrated circuits due to their zero leakage and steep subthreshold properties. In this paper, we explore the implementation of the $\mathrm{I} / \mathrm{O}$ interface circuits, namely the analog-todigital converter (ADC) and the digital-to-analog converter (DAC), with back-end-of-line (BEOL) NEM relays. The proposed design for the ADC comparators eliminates the need for reference generation by utilizing a bank of NEM relays with different pullin voltages. The decoder and encoder of both data converters are optimized by using a minimum number of devices and exclusive, non-leaking paths for each input and output code. As a result, while the sampling rate and operation frequency of the relaybased converters are inherently lower than CMOS counterparts due to mechanical nature of operation, the proposed data converters can achieve at least one order of magnitude lower energy consumption, which makes them appealing alternatives for ultra-low power VLSI and Internet of Things (IoT) applications.
\end{abstract}

Keywords-BEOL NEM relay, Ultra-low power VLSI design, Analog-to-Digital converter, Digital-to-Analog converter

\section{INTRODUCTION}

The NEM relays have been studied and demonstrated extensively in diverse VLSI applications such as logic gates, arithmetic blocks, memory cells and power management [1-5]. The potential of NEM relay technology for low power I/O units and data converters have also been investigated [1-3]. However, no complete circuit implementation of data converters can be found in the previous works, and the proposed schemes suffer from significant static power dissipation.

Recently a CMOS-compatible, vertical NEM relay that utilizes BEOL process and airgap technology has been proposed $[6,7]$. In this work, we use a similar structure and customize it to demonstrate a novel implementation of all-NEM relay 4-bit data converters. By minimizing the number of devices for each block, removing the current leakage path and optimizing the operational voltage of relays, the proposed design in this paper can reduce the energy per operation cost significantly. We use Coventor MEMS+ [8] to investigate the electromechanical behavior of the relays and Cadence Virtuoso Spectre for the circuit implementation and simulation with a Verilog-A behavioral description model that we have developed for the BEOL relay.

The rest of this paper is organized as follows. The device structure, operation principles and methods for tuning and customizing the device behavior are presented in section II. The architecture and simulation results of the proposed ADC and DAC, and their corresponding decoder/encoders, are discussed
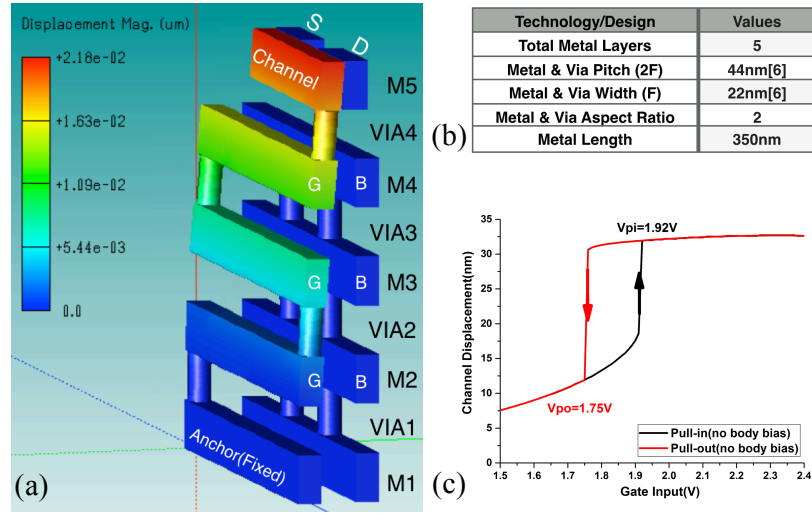

Fig. 1. BEOL NEM relay (adapted from [6]). (a) 3D illustration of the relay under pull-in state. (b) Technological specifications. (c) Pull-in and pull-out behaviors.

in sections III and IV, respectively. A short discussion on the results and main conclusions are presented in section $\mathrm{V}$.

\section{Device Strcuture, Operation AND TUNING}

\section{A. Device Structure and Operation}

Similar to the CMOS transistor, the NEM relay, depicted in Fig. 1(a), has four terminals: a moving gate beam (G), and fixed source (S), drain (D) and body (B) electrodes, all made of aluminum. The gate spans from M1 to M4 layers and the channel is on the M5 layer. M1 is fixed as an anchor. The VIA between M4 and M5 is inter-metal dielectric made of silicon dioxide and prevents the flow of current from the gate to the channel. The technological specifications are listed in Fig. 1(b).

When a voltage difference is applied between the gate (the free moving beam) and body electrodes, the beam will bend towards the body due to the electrostatic force generated in between. If this force is strong enough to overcome the spring restoring force, the beam "pulls in," and the channel connects the source and the drain. Now if we reduce the applied voltage, at a certain point, the spring restoring force becomes larger than the electrostatic force and surface contact forces (including Van der Waals' force between atoms and capillary force due to a certain humidity in the air [9]), thus the beam "pulls out". As a consequence, the operation is inherently hysteretic, a fact that is reflected by a $0.17 \mathrm{~V}$ difference between the pull-out voltage $\left(\mathrm{V}_{\mathrm{PO}}\right)$ and pull-in voltage $\left(\mathrm{V}_{\mathrm{PI}}\right)$, as illustrated in Fig. 1(c).

\section{B. Tuning the Pull-in Voltage}

The design of ADC comparators, explained in the next section, requires relays with different $V_{\text {PI. The }}$ are a few methods for tuning the spring constant of the beam and thus the

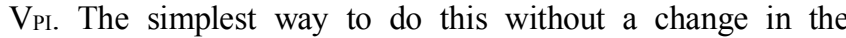
dimensions and area of the device is by adjusting the inter-metal 
TABLE I. VIA ARRANGEMENTS FOR V VI TUNING

\begin{tabular}{|c|c|c|c|c|c|c|c|c|}
\hline $\mathrm{V}_{\mathrm{PI}}(\mathrm{V})$ & 1.92 & 2.02 & 2.12 & 2.22 & 2.32 & 2.42 & 2.52 & 2.62 \\
\hline VIA Arrangements & 151 & 515 & 154 & 525 & 144 & 412 & 541 & 355 \\
\hline $\mathrm{V}_{\mathrm{PI}}(\mathrm{V})$ & 2.72 & 2.82 & 2.92 & 3.02 & 3.12 & 3.22 & 3.32 & \multicolumn{1}{|c}{} \\
\hline VIA Arrangements & 244 & 522 & 431 & 233 & 224 & 444 & 333 & \multicolumn{1}{|c}{} \\
\cline { 1 - 6 } & & & & & &
\end{tabular}

VIA locations. By doing that, the $\mathrm{V}_{\mathrm{PI}}$ can be tuned by changing the effective length of the spring ( $\left.\mathrm{L}_{\mathrm{eff}}\right)$. The serpentine-shaped gate in Fig. 1 (a) represents the largest $L_{\text {eff, }}$ hence provides the minimum stiffness and spring constant. Therefore, minimum $V_{P I}$ is obtained with this structure. In this work, we achieve the $V_{P I}$ tuning by varying the location of VIA1 to VIA3 along the beam. We assign the VIA locations to five points along the metal beam, including both ends, to observe distinct shifts in the VPI. We call the possible locations from far left to far right as position " 1 " to "5 as annotated in Fig. 2(a), and the full arrangement is represented by a three-digit number representing the position of VIA1 to VIA3. As an example, the structure in Fig. 1(a) has the VIA arrangements of " $151 "$.

As the comparator bank of the 4-bit flash ADC in this paper requires 15 devices with uniform changes on $\mathrm{V}_{\mathrm{PI}}$, the tuning method stated above is implemented on the structure. Fig. 2 displays four examples of the device variations and the resulting VPI. TABLE I summarizes simulated VPI for all desired devices.

\section{Tuning the Operating Voltage with Body Biasing}

The NEM relay is actuated by the electrostatic force generated between the moving gate and the fixed body, conventional way of implementing this is applying $\mathrm{V}_{\mathrm{PI}}$ on the gate while the body is grounded. However, biasing the body with a negative voltage while keeping the voltage difference identical can lower the required input voltage on the gate. When the body is biased at $-V_{P O}$, the relay can be switched on with only ( $V_{P I}-$ $\mathrm{VPO}_{\mathrm{PO}}$ ). If this method is applied to the structure in Fig. 1(a), it results in a $V_{P I}$ of $0.17 \mathrm{~V}$ and $V_{P O}$ of $0 \mathrm{~V}$, which is the minimum operating voltage achievable as described in Fig. 3(a).

In addition to more freedom on the circuit design, this method also saves more than one order of magnitude dynamic energy for most cases, as a direct result of operating in lower voltage domain. A practical operating point to ensure safe functioning is suggested in Fig. 3(b) with the simulated results from Cadence Virtuoso pointing out an abrupt switching behavior in the I-V curve.

\section{BEOL NEM RELAY FLASH ADC}

An overview of the ADC design is illustrated in Fig. 4. The analog input $\left(\mathrm{V}_{\mathrm{A}}\right)$ goes through a standard sample and hold $(\mathrm{S} / \mathrm{H})$ sub-circuit[1], then it is picked up by the comparators. The
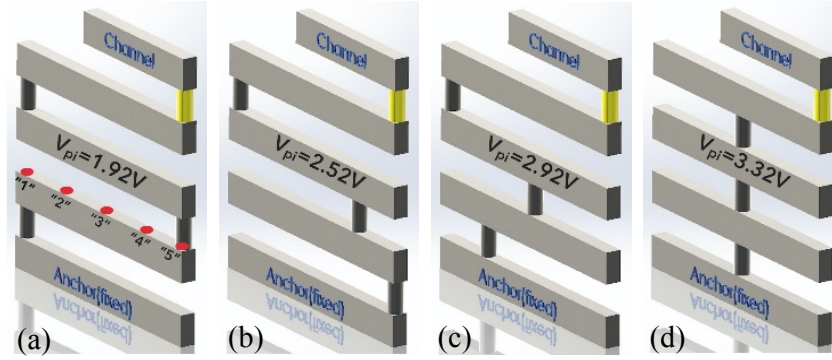

Fig. 2. Showcase of VIA arrangements to obtain different $V_{\text {PI. (a) Arrangement }}$ "151" (b) Arrangement "541" (c) Arrangement "231" (d) Arrangement "333".
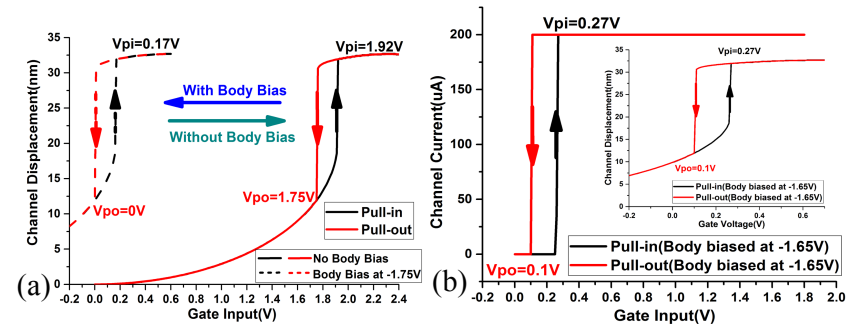

Fig. 3. (a) Body biasing scheme. (b) Practical operating point adopted, the inset shows the corresponding pull-in and pull-out behavior.

outputs of comparators are then converted from thermometer coded (TC) to standard binary output. The comparators utilize a variety of relay structures mentioned in TABLE I. The details of the design will be discussed in this section.

\section{A. Design Methodology for ADC Core}

The schematic block diagram in Fig. 4 exhibits the architecture of the all-relay flash ADC. The body of the comparators and the decoder is biased with a negative voltage, $\mathrm{V}_{\mathrm{NEG}}$, to enable the operation of the circuits with an independent low voltage supply (VLOW) thus reducing the total energy consumption. The control signals used in the circuit include the sample signal (SAMP) and the hold signal (HOLD) to guarantee a valid and fixed analog input for the comparators, $V_{\text {HOLD, during }}$ each conversion. $V_{\text {HOLD, }}$ the shared input among all comparators, is effectively compared with the $V_{\text {PI }}$ of various relays simultaneously, deciding the switching behavior of those relays. Each comparator's output $\left(\mathrm{OUT}_{\mathrm{n}}\right)$ is first pre-discharged to GND when the evaluation signal (EVAL) is 0 . When EVAL becomes 1, OUT $_{n}$ is evaluated (charged) to VLow only if VHOLD is large enough to turn on that specific comparator relay (with a unique pull-in voltage of $\mathrm{VPI}, \mathrm{n})$, otherwise $\mathrm{OUT}_{\mathrm{n}}$ remains 0 .

Note that only the comparators utilize relay structures with

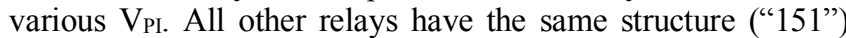
which exhibits the minimum $\mathrm{V}_{\mathrm{PI}}$ and optimal energy and speed.

\section{B. 15-line to 4-line TC to Binary Relay Decoder}

The 15-bit TC output needs to be converted to its standard 4bit binary equivalent. We designed a relay-based decoder that generates the final output $D_{3} D_{2} D_{1} D_{0}$ based on the truth table in Fig. 5(a). The TC output, OUT in Fig. 5(a), contains consecutive 1 's and 0 's. This property can be used to simplify the decoder with a methodology shown in Fig. 5(b). The implementations

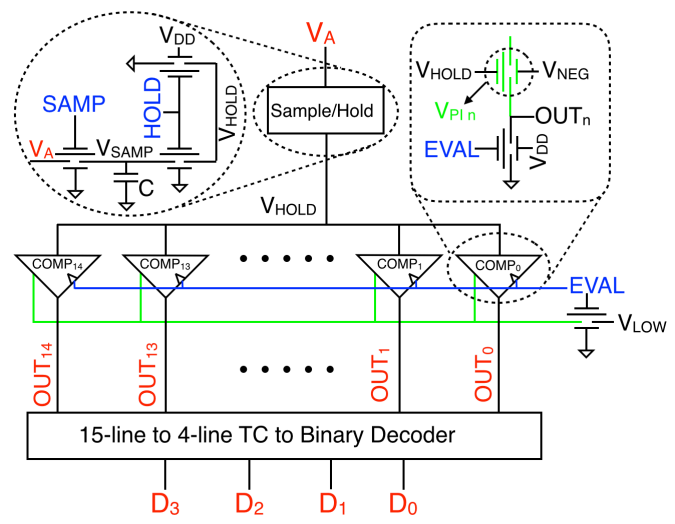

Fig. 4. Design flow of the proposed ADC with circuit implementation illustration of sample/hold block and comparators. 
(a)

\begin{tabular}{|c|c|c|c|c|c|c|}
\hline OUT & $\mathbf{D}_{3}$ & $D_{2}$ & $\mathrm{D}_{1}$ & $\mathbf{D}_{0}$ & \multirow{3}{*}{\multicolumn{2}{|c|}{$\mathrm{D}_{0}=\mathrm{OUT}_{0} \oplus \mathrm{OUT}_{1} \oplus \ldots \oplus \mathrm{OUT}_{14}$}} \\
\hline $\begin{array}{l}00000000000000000 \\
000000000000001\end{array}$ & $\frac{0}{0}$ & $\frac{0}{0}$ & $\frac{0}{0}$ & \begin{tabular}{l|l}
0 \\
1
\end{tabular} & & \\
\hline 000000000000011 & 0 & 0 & 1 & 0 & & \\
\hline$\frac{0000000000000111}{0.000000000111}$ & 0 & 0 & $a_{0}$ & 1 & & $\left.\mathrm{D}_{1}=\overline{\mathrm{OUT}} \cdot \boldsymbol{\bullet} \overline{\mathrm{OUT}} \cdot \boldsymbol{\mathrm { OUT }}+\mathrm{OUT} \cdot \boldsymbol{\mathrm { OUTT }}\right)+$ \\
\hline 0000000000000111111 & 0 & $\frac{1}{1}$ & 0 & 1 & & 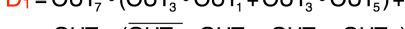 \\
\hline 000000000001111111 & 0 & 1 & 1 & 0 & & $\mathrm{OUT}_{7} \cdot\left(\mathrm{OUT}_{11} \bullet \mathrm{OUT}_{9}+\mathrm{OUT}_{11} \bullet \mathrm{OUT}_{13}\right)$ \\
\hline 00000000011111111 & 0 & 1 & 1 & 1 & & \\
\hline $\begin{array}{l}000000011111111 \\
000000111111111\end{array}$ & 1 & 0 & 0 & 0 & & \\
\hline 00000000111111111111 & 1 & 0 & 0 & T & & $\mathrm{D}_{2}=\mathrm{OUT}_{7} \cdot \mathrm{OUT}_{3}+\mathrm{OUT}_{7} \cdot \mathrm{OUT}_{11}$ \\
\hline 000011111111111 & 1 & 0 & 1 & 1 & & \\
\hline 000111111111111 & 1 & 1 & 0 & 0 & & $\mathrm{D}_{3}=\mathrm{OUT}_{7}$ \\
\hline 0011111111111111 & 1 & 1 & 0 & & & \\
\hline 011111111111111111 & \begin{tabular}{|c|}
1 \\
1
\end{tabular} & 1 & $\frac{1}{1}$ & $\frac{0}{1}$ & (b) & \\
\hline
\end{tabular}

Fig. 5. (a) Truth table of the 15-bit to 4-bit decoder. (b) Design methodology. are demonstrated in Fig. 6. The circuit is optimized to implement the decoder with as few as 68 NEM relays and only incurs one mechanical delay $(\sim 20 \mathrm{~ns})$, the total delay of the ADC is approximately two mechanical time constants. Fig. 7 reveals the Spectre simulation results of the NEM relay ADC with a sampling rate of $2.5 \mathrm{MHz}$. The TC output plots are replaced with binary blocks for better visualization.

The total number of relays used to implement the ADC is 102 , and the energy consumed during each conversion step is $0.74 \mathrm{fJ}$ on average. This low energy consumption behavior is attributed to the absence of static direct current, e.g., voltage reference level provided by resistor string in traditional flash ADCs, and low swing of operational voltage $\left(\mathrm{V}_{\text {LOW }}\right)$. The energy/op consumed in this circuit shows more than $96.6 \%$ reduction compared to the flash relay ADC proposed in [1]. The comparison between this work and state of the art relay ADC as well as a few CMOS ADCs are listed in TABLE II. The figure of merit used in this paper is Walden FOM [13], FOM= Power/ $\left(2^{\mathrm{n} *}\right.$ Sampling Rate), where $\mathrm{n}$ is the resolution of the ADC.

\section{BEOL NEM RELAY DAC}

In this section, we describe the implementation of all-relay DAC. Fig. 8(a) illustrates the schematic of the proposed DAC. Here we follow a design flow that is the reverse of the ADC, where we encode the 4-bit input into a 15-bit TC output via a dedicated relay encoder and then convert it to the final analog output using a bank of relay buffers. Each buffer is driven by one of the TC input lines and followed by a terminating resistor. This topology can generate the output voltage with a fixed step increment of $\mathrm{V}_{\mathrm{LOW}} / \mathrm{N}$, where $\mathrm{N}=2^{\mathrm{k}}-1$ and $\mathrm{k}$ is the bit resolution
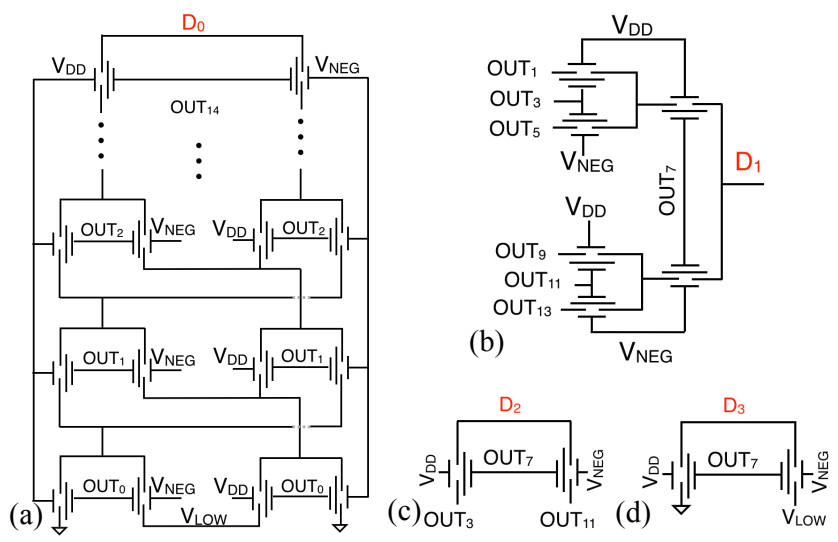

Fig. 6. The circuit implementation of the all-relay TC to binary decoder. Only one mechanical delay is introduced by this block. (a) $D_{0}$ bit. (b) $D_{1}$ bit. (c) $D_{2}$ bit. (d) $D_{3}$ bit, a buffer is introduced for an identical delay with other bits.

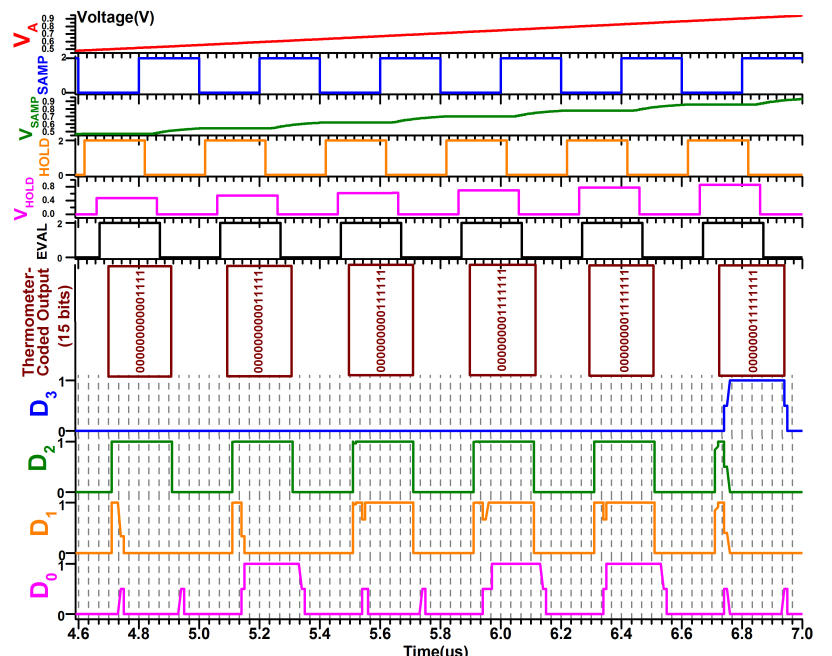

Fig. 7. Simulation results of the NEM relay-based $A D C, V_{L O W}=1 \mathrm{~V}, V_{D D}=$ $2 \mathrm{~V}, \mathrm{~V}_{\mathrm{NEG}}=-1 \mathrm{~V}, \mathrm{C}=500 \mathrm{fF}$.

of the DAC. The analog output voltage $\mathrm{V}_{\mathrm{A}}$ is determined by the number of 1 's in the encoder's output, or $\mathrm{V}_{\mathrm{A}}=(D / 15) * \mathrm{~V}_{\mathrm{LOW}}$.

We use the reverse of the truth table in Fig.5(a) to extract the expressions for the binary to TC encoder. The algorithm is summarized in Fig. 8(b). Since many of the TC bits share similar expressions, we share some relays in the main path for multiple outputs. The constructed circuit is shown in Fig. 8(c), in which 11 out of 15 outputs reuse some relays, resulting in minimized device count of 84 . The devices used in the DAC have identical

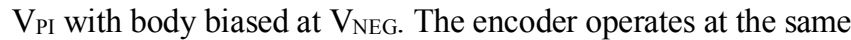
independent $\mathrm{I} / \mathrm{O}$ voltage $\mathrm{V}_{\text {LOw }}$ as well for energy saving.

Similar to the ADC, the full DAC circuit incurs two mechanical delays, so we operate it at the same frequency as the ADC. The simulation results are shown in Fig. 9, where the analog output accurately represents the digital input. The DAC consumes $5.82 \mathrm{fJ} /$ conversion while the encoder itself consumes a negligible amount of energy of $0.03 \mathrm{fJ} /$ conversion. The comparison of the results with state of the art CMOS DACs is summarized in TABLE III.

\section{CONCLUSION}

This paper introduces the design methodology for all-NEM relay based data converters, customized for predictive vertical relays fabricated in BEOL process. The reference generation unit traditionally implemented by resistor strings or similar methods in flash ADCs has been removed by tuning the operational voltage of each NEM relay in the comparator bank. The decoder of the ADC and the encoder of the DAC are TABLE II. COMPARISONS OF THE ADCS

\begin{tabular}{|c|c|c|c|c|c|}
\hline & $\begin{array}{c}\text { VLSI'13 } \\
{[10]}\end{array}$ & $\begin{array}{c}\text { TVLSI'14 } \\
{[11]}\end{array}$ & $\begin{array}{c}\text { VLSI'17 } \\
{[12]}\end{array}$ & $\begin{array}{c}\text { ICCAD' } \\
08[1]\end{array}$ & This Work \\
\hline $\begin{array}{c}\text { Device } \\
\text { Technology }\end{array}$ & $\begin{array}{c}\text { CMOS } \\
/ 22 \mathrm{~nm}\end{array}$ & $\begin{array}{c}\text { CMOS } \\
/ 65 \mathrm{~nm}\end{array}$ & $\begin{array}{c}\text { CMOS } \\
/ 65 \mathrm{~nm}\end{array}$ & $\begin{array}{c}\text { NEM Relay } \\
/ 90 \mathrm{~nm}\end{array}$ & $\begin{array}{c}\text { NEM Relay } \\
/ 22 \mathrm{~nm}\end{array}$ \\
\hline Architecture & $\Delta \Sigma$ & Flash & Flash & Flash & Flash \\
\hline $\begin{array}{c}\text { Resolution } \\
\text { (bit) }\end{array}$ & 8 & 4 & 8 & 6 & 4 \\
\hline $\begin{array}{c}\text { Sampling } \\
\text { Rate }\end{array}$ & $240 \mathrm{MS} / \mathrm{s}$ & $10 \mathrm{GS} / \mathrm{s}$ & $2 \mathrm{GS} / \mathrm{s}$ & $10 \mathrm{MS} / \mathrm{s}$ & $2.5 \mathrm{MS} / \mathrm{s}$ \\
\hline $\begin{array}{c}\text { Power (mW) } \\
\text { FOM } \\
\text { (fJ/Conv.step) }\end{array}$ & 12.7 & 104 & 21 & $3.5 \times 10^{-3}$ & $2.97 \times 10^{-5}$ \\
\hline \multicolumn{1}{|c|}{259.6} & 880 & 109.4 & 5.5 & 0.74 \\
\hline
\end{tabular}




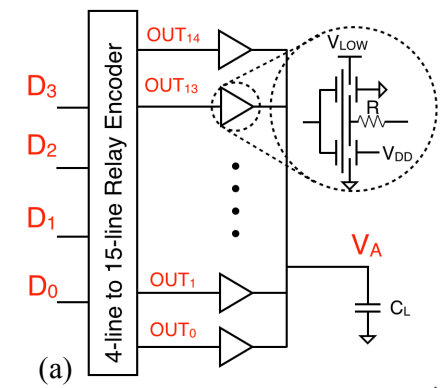

$\mathrm{OUT}_{0}=\mathrm{D}_{3}+\mathrm{D}_{2}+\mathrm{D}_{1}+\mathrm{D}_{0}$ $\mathrm{OUT}_{1}=\mathrm{D}_{3}+\mathrm{D}_{2}+\mathrm{D}_{1}$

$\mathrm{OUT}_{2}=\mathrm{D}_{3}+\mathrm{D}_{2}+\mathrm{D}_{1} \cdot \mathrm{D}_{0}$

$\mathrm{OUT}_{3}=\mathrm{D}_{3}+\mathrm{D}_{2}$

$\mathrm{OUT}_{4}=\mathrm{D}_{3}+\mathrm{D}_{2} \cdot \mathrm{D}_{1}+\mathrm{D}_{2} \cdot \mathrm{D}_{0}$

$\mathrm{OUT}_{5}=\mathrm{D}_{3}+\mathrm{D}_{2} \cdot \mathrm{D}_{1}$

$\mathrm{OUT}_{6}=\mathrm{D}_{3}+\mathrm{D}_{2} \cdot \mathrm{D}_{1} \cdot \mathrm{D}_{0}$

$\mathrm{OUT}_{7}=\mathrm{D}_{3}$

$\mathrm{OUT}_{8}=\mathrm{D}_{3} \cdot\left(\mathrm{D}_{2}+\mathrm{D}_{1}+\mathrm{D}_{0}\right)$

$\mathrm{OUT}_{9}=\mathrm{D}_{3} \cdot\left(\mathrm{D}_{2}+\mathrm{D}_{1}\right)$

$\mathrm{OUT}_{10}=\mathrm{D}_{3} \cdot\left(\mathrm{D}_{2}+\mathrm{D}_{1}\right) \cdot\left(\mathrm{D}_{2}+\mathrm{D}_{0}\right)$

$\mathrm{OUT}_{11}=\mathrm{D}_{3} \cdot \mathrm{D}_{2}$

$\mathrm{OUT}_{12}=\mathrm{D}_{3} \cdot \mathrm{D}_{2} \cdot\left(\mathrm{D}_{1}+\mathrm{D}_{0}\right)$

$\mathrm{OUT}_{13}=\mathrm{D}_{3} \cdot \mathrm{D}_{2} \cdot \mathrm{D}_{1}$

(b) $\mathrm{OUT}_{14}=\mathrm{D}_{3} \cdot \mathrm{D}_{2} \cdot \mathrm{D}_{1} \cdot \mathrm{D}_{0}$
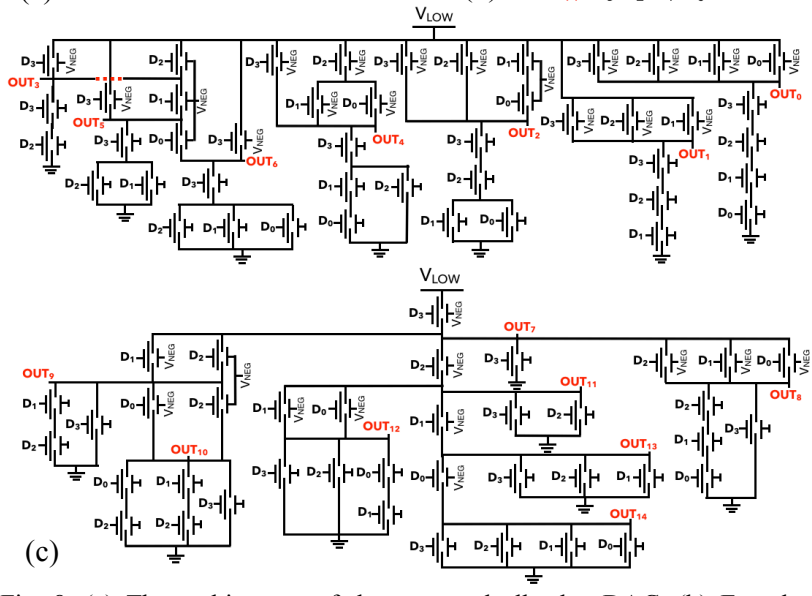

Fig. 8. (a) The architecture of the proposed all-relay DAC. (b) Encoder output expressions and (c) encoder circuit implementation.

optimized to remove recurring current paths and achieve the minimum number of devices for each function. The operational voltage of the relays has been reduced to a minimum which is

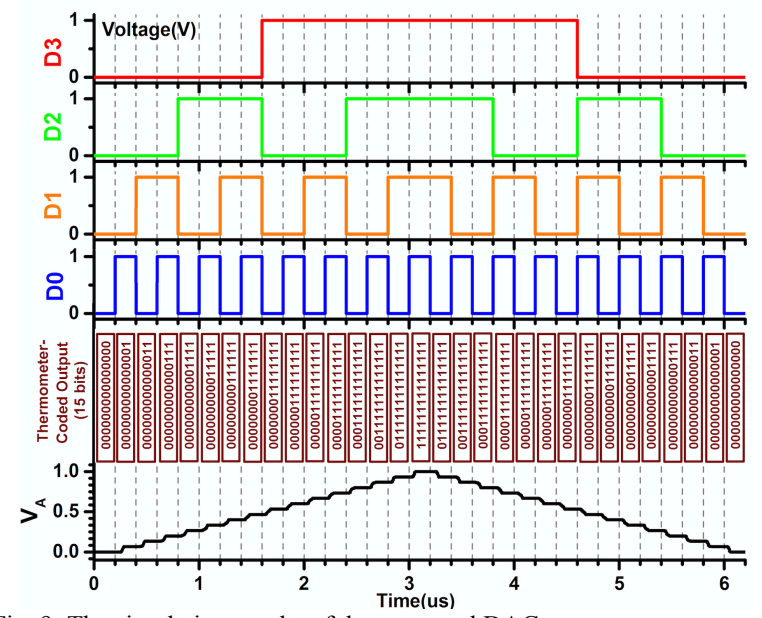

Fig. 9. The simulation results of the proposed DAC.

TABLE III. COMPARISONS OF THE DACS

\begin{tabular}{|c|c|c|c|c|c|}
\hline & {$[14]$} & {$[15]$} & {$[16]$} & {$[17]$} & This Work \\
\hline $\begin{array}{c}\text { Device } \\
\text { Technology }\end{array}$ & $\begin{array}{c}\text { CMOS } \\
/ 65 \mathrm{~nm}\end{array}$ & $\begin{array}{c}\text { CMOS } \\
/ 90 \mathrm{~nm}\end{array}$ & $\begin{array}{c}\text { CMOS } \\
/ 40 \mathrm{~nm}\end{array}$ & $\begin{array}{c}\text { CMOS } \\
/ 0.13 \mu \mathrm{m}\end{array}$ & $\begin{array}{c}\text { NEM Relay } \\
/ 22 \mathrm{~nm}\end{array}$ \\
\hline $\begin{array}{c}\text { Resolution } \\
\text { (bit) }\end{array}$ & 4 & 5 & 6 & 6 & 4 \\
\hline Sampling Rate & $10 \mathrm{GS} / \mathrm{s}$ & $2 \mathrm{GS} / \mathrm{s}$ & $4 \mathrm{GS} / \mathrm{s}$ & $2.7 \mathrm{GS} / \mathrm{s}$ & $2.5 \mathrm{MS} / \mathrm{s}$ \\
\hline Power (mW) & 30 & 12 & 13 & 5.4 & $1.064 \times 10^{-3}$ \\
\hline $\begin{array}{c}\text { FOM } \\
\text { (fJ/Conv.step) }\end{array}$ & 190 & 187.5 & 50.8 & 31 & 5.82 \\
\hline
\end{tabular}

enforced by the inherent hysteresis between $V_{P I}$ and $V_{P O}$. The energy efficiency is significantly improved for both DAC and $\mathrm{ADC}$ as a result of these optimizations. While the conversion speed of relay-based data converters is considerably lower than the CMOS counterparts due to the mechanical nature of their operation, the superior energy per conversion proves the feasibility of this technology for ultra-low power applications with modest performance requirement, such as IoT and biomedical applications.

\section{ACKNOWLEDGMENT}

The authors are grateful to Professor Tsu-Jae King Liu of University of California, Berkeley for the valuable discussions.

\section{REFERENCES}

[1] F. Chen, H. Kam, D. Marković, et al. "Integrated circuit design with NEM relays", in International Conference on Computer-Aided Design (ICCAD), 2008, pp. 750-757.

[2] M.Spencer, F.Chen, C.C.Wang, et al. "Demonstration of integrated micro-electro-mechanical relay circuits for VLSI applications", IEEE Journal of Solid-State Circuits, 2011, vol. 46, no. 1, pp. 308-320.

[3] F.Chen, M.Spencer, R.Nathaneal, et al. "Demonstration of integrated micro-electro- mechanical switch circuits for VLSI applications", in International Solid-State Conference (ISSCC), 2010, pp. 150-151.

[4] H. Fariborzi, M. Spencer, V. Karkare, et al. "Analysis and demonstration of NEM- relay power gating", in Custom Integrated Circuits Conference (CICC) 2010, pp. 1-4.

[5] H. Fariborzi, F. Chen, R. Nathaneal, et al. "Relay do not leak-CMOS does", in Design Automation Conference (DAC), 2013, pp. 1-4.

[6] N. Xu, J. Sun, I-R. Chen, et al. "Hybrid CMOS/BEOL-NEMS technology for ultra-low power IC applications", in International Electron Device Meeting (IEDM), 2014, pp. 28.8.1-28.8.4.

[7] A. Peschot, C. Qian and T.-J. K. Liu. "Nanoelectromechanical Switches for low- power digital computing", Michromachines, 2015, vol. 6, no. 8, pp. 1046-1065.

[8] "MEMS+ 6.300 Reference", March 2018, Coventor MEMS+.

[9] H. F. Dadgour, M. M. Hussain, A. Cassell, et al. "Impact of scaling on the performance and reliability degradation of metal-contacts in NEMS devices", in International Reliability Physics Symposium (IRPS), 2011, pp. 3D.3.1-3D.3.10

[10] C. C. Lee, E. Alpman, S. Weaver, et al. "A 66dB SNDR 15MHz BW SAR Assisted $\triangle \Sigma$ ADC in 22nm Tri-gate CMOS" in Symposium on VLSI Circuits, 2013, pp. C64-C65.

[11] Y. Xu, L. Belostotski, and J. W. Haslett. "A 65-nm CMOS 10-GS/s 4-bit Background- Calibrated Noninterleaved Flash ADC for Radio Astronomy", IEEE Transactions on Very Large Scale Integration (VLSI) Systems, 2014, vol. 22, no. 1, pp. 2316- 2325.

[12] J-I. Kim, D.-R. Oh, D.-S. Jo, et al. "A 65 nm CMOS 7b 2 GS/s $20.7 \mathrm{~mW}$ Flash ADC With Cascaded Latch Interpolation", IEEE Journal of SolidState Circuits, 2015, vol. 50, no. 10, pp. 2319-2330.

[13] B. Murmann, “ADC Performance Survey 1997-2017”, [Online]. Available: https://web.stanford.edu/ murmann/adcsurvey.html

[14] F. N. U. Juanda, W. Shu, and J. S. Chang. "A 10GS/s 4-bit single-core digital-to-analog converter for cognitive ultra-widebands", IEEE Trans. Circuits Syst. II, Exp. Briefs, 2017, vol. 64, no. 1, pp. 16-20.

[15] X. Wu, and M. Steyaert. "A 90nm CMOS 5-bit 2GS/s DAC for UWB Transceivers", in International Conference on Ultra- Wideband (ICUWB), 2010, pp.1-4.

[16] L. Zhao, J. He, and Y. Cheng. "A 6bit 4GS/s Current-steering Digital-toAnalog Converter in 40nm CMOS with Adjustable Bias and DfT Block", in International Conference on ASIC (ASICON), 2015, pp.1-4.

[17] R.-L. Chen, H.-W. Ting, and S.-J. Chang. "Six-bit 2.7-GS/s 5.4-mW Nyquist complementary metal-oxide semiconductor digital-to-analogue converter for ultra-wideband transceivers", IET Circuits, Devices \& Systems, 2012, vol. 6, no. 2, pp. 95-102. 